\title{
Suppressed primary osteoblast functions on nanoporous titania surface
}

\author{
Lingzhou Zhao, ${ }^{1}$ Shenglin Mei, ${ }^{2}$ Wei Wang, ${ }^{2}$ Paul K. Chu, ${ }^{3}$ Yumei Zhang, ${ }^{2}$ Zhifen $\mathbf{W u}^{1}$ \\ ${ }^{1}$ Department of Periodontology and Oral Medicine, School of Stomatology, The Fourth Military Medical University, \\ No. 145 West Changle Road, Xi'an 710032, China \\ ${ }^{2}$ Department of Prosthetic Dentistry, School of Stomatology, The Fourth Military Medical University, \\ No. 145 West Changle Road, Xi'an 710032, China \\ ${ }^{3}$ Department of Physics and Materials Science, City University of Hong Kong, Tat Chee Avenue, Kowloon, Hong Kong, China
}

Received 27 August 2009; revised 3 May 2010; accepted 24 June 2010

Published online 4 November 2010 in Wiley Online Library (wileyonlinelibrary.com). DOI: 10.1002/jbm.a.32918

\begin{abstract}
Titiania nanotubes have large potential in medical implant applications but their tissue compatibility is still controversial. Considering that the biological behavior of primary osteoblasts is closer to the in vivo situation than other common cell lines, we investigate the response of primary osteoblasts on anodized nanotextured titania surfaces. Two nanotextured surface morphologies, namely the $5 \mathrm{~V}$ anodized surface with a pore diameter of $25 \mathrm{~nm}$ and the $20 \mathrm{~V}$ anodized surface with a tube diameter of $80 \mathrm{~nm}$ are chosen for this study. Initial cell adhesion is not obviously affected by the anodized surfaces. With the exception of slightly higher intracellular alkaline phosphatase activity and more extracellular
\end{abstract}

matrix deposition, cell growth, and cell differentiation represented by the expressions of osteogenesis-related genes are impaired on both anodized surfaces. This may be attributed to the compromised focal contact formation on the anodized surfaces. The difference in the phenotypes of the primary osteoblasts and the osteoblastic cell lines may partly account for the controversy in osteoblast cytocompatibility on titania nanotubes. (c) 2010 Wiley Periodicals, Inc. J Biomed Mater Res Part A: 96A: 100-107, 2011.

Key Words: nanotopography, primary osteoblast, cell adhesion, alkaline phosphatase activity, real-time PCR

\section{INTRODUCTION}

The surface topography on biomedical implants plays a critical role in osseointegration and subsequent clinical success. Previous studies have shown that micrometer or submicrometer surfaces can enhance bone cell functions and promote implant osseointegration. Since bone tissues are composed of nanofibered hydroxyapatite dispersed in a mostly collagen matrix, it has been suggested that a nanotopography may more efficiently facilitate interactions with bone cell. ${ }^{1-3}$

Many types of nanotopography have been adopted to study cellular response to the nanotextured surfaces, among which the titania nanotubular surface has drawn much attention. Vertically oriented and highly ordered titania nanotube arrays can be fabricated easily by simple anodization and the nanotube dimensions can be controlled by adjusting the experimental parameters. ${ }^{4-6}$ Titania nanotubes can induce the growth of nanodimensioned hydroxyapatite ${ }^{7}$ and it has been reported that titania nanotubes with a suitable size may enhance the functions of bone cells as well. ${ }^{6,8-10}$ Besides, the nanotubes can serve as carriers for drugs such as growth factors ${ }^{11}$ or bacteriocides. ${ }^{12}$ Titania nanotubes also have a smaller Young's modulus of $\sim 36-43 \mathrm{GPa}^{13}$ which is closer to that of bones compared with titanium. It may prevent bone resorption caused by stress shielding.
Although titania nanotubes have many favorable properties as bone implants, the biological performance of titania nanotubes is still not well understood. It has been reported that titania nanotubes with different sizes of 30-100 nm, ${ }^{6}$ $50 \mathrm{~nm}^{10} 70 \mathrm{~nm},{ }^{8,11}$ and $80 \mathrm{~nm}^{9}$ enhance osteoblast cell functions ${ }^{6,8-11}$ and in vivo bone bonding. ${ }^{14}$ However, Park et al. have obtained contrary results that nanotubes with a smaller tube size $(15 \mathrm{~nm})$ strongly enhances cell activities, while cell functions which degrade with increasing tube size are severely impaired on nanotube layers with a tube diameter larger than $50 \mathrm{~nm} .{ }^{4,5}$ We believe that the different cell types used in these experiments may be one of the reasons for the controversy on the cytocompatibility of titania nanotubes. ${ }^{15,16}$ Actually, Peng et al.'s work may support this hypothesis, showing that endothelial cell and vascular smooth muscle responds oppositely to titania nanotubes. ${ }^{17}$ We have also observed different response of fibroblast, epithelium and osteoblast to titania nanotubes in our ongoing experiments (unpublished data).

In nearly all the studies on the beneficial effects of titania nanotubes with different sizes on osteoblast/implant interactions, some osteoblastic cell strains such as MC3T3$\mathrm{E} 1,{ }^{6,8,9,18}$ human osteoblast-like cell, ${ }^{11}$ and human osteoblastic precursor cell line ${ }^{10}$ are used, because they are relatively easy to obtain and handle and generally offer better reproducibility

Correspondence to: Y. Zhang; e-mail:wqtzym@fmmu.edu.cn or Z.Wu; e-mail: wzfwxy@fmmu.edu.cn

Contract grant sponsor: National Natural Science Foundation of China; contract grant number: 30672347

Contract grant sponsor: Hong Kong Research Grants Council (RGC)

Contract grant sponsor: General Research Fund (GRF); contract grant number: CityU 112307 

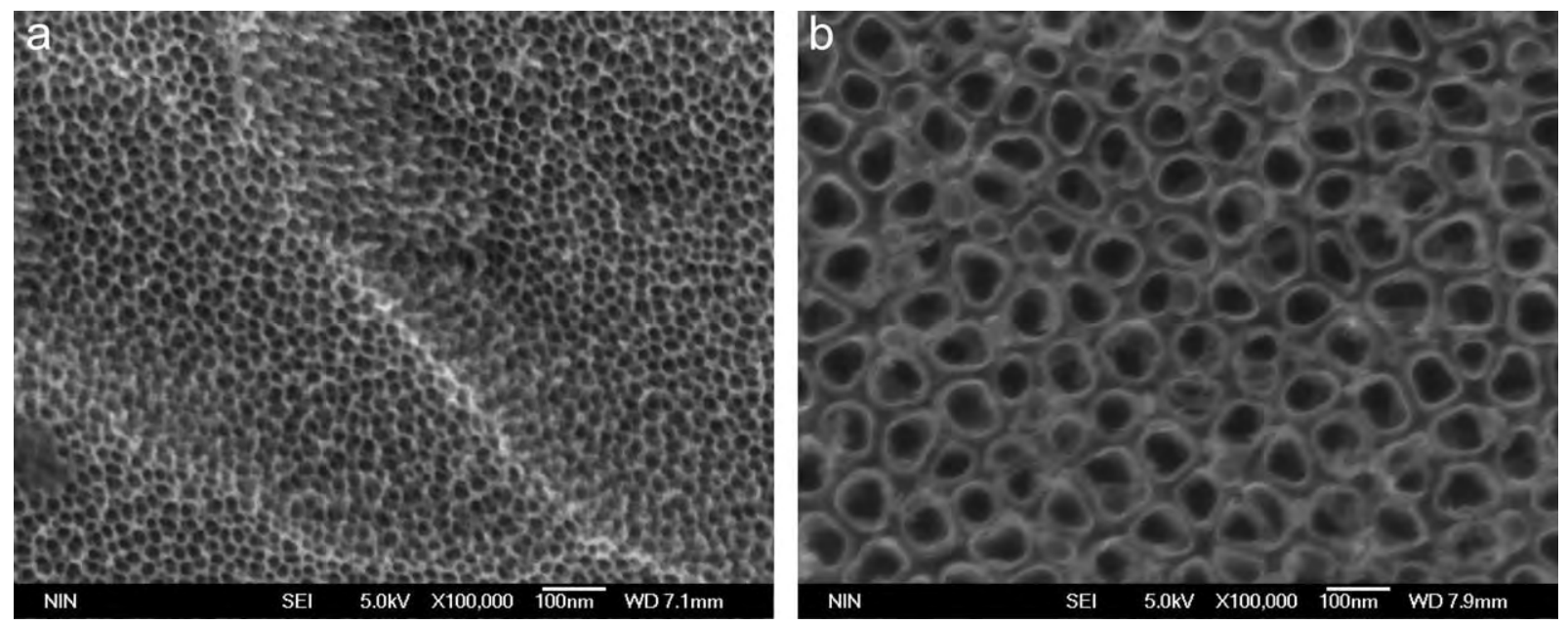

FIGURE 1. The anodized nanotextured surfaces formed at $5 \mathrm{~V}$ (a) and $20 \mathrm{~V}$ (b), respectively.

of results than primary cell. However, the biological characteristics of cell strains are very different from those of cells in vivo. In this study, to study the osseointegration ability of titania nanotubes, we investigate the response of primary osteoblasts whose biological behavior is closer to that of cells in vivo. Our experiments are aimed at providing a better understanding of the cytocompatibility of titania nanotubes to hopeful resolve the current controversy.

\section{MATERIALS AND METHODS}

Specimen preparation

Pieces of cp titanium with $99.8 \%$ purity $(10 \times 10 \times$ $1 \mathrm{~mm}^{3}$ ) were used as substrates for anodization. After polishing and ultrasonic cleaning, the substrates were anodized for $30 \mathrm{~min}$ in $0.5 \mathrm{wt} \%$ hydrofluoric acid using a DC power supply and a platinum electrode serving as the cathode. Two typical kinds of nanotextured surface were formed at different anodizing potentials, namely the $5 \mathrm{~V}$ anodized surface with a nanonet-like texture and the $20 \mathrm{~V}$ anodized surface with a nanotubular texture (Fig. 1). The polished surface $(\mathrm{P})$ was used to observe the cellular behavior with the cell culture polystyrene (C) serving as the control. Immediately after anodization, the samples were rinsed with deionized water. After ultrasonic cleaning, the samples were sterilized by ultraviolet irradiation for $30 \mathrm{~min}$ on each side before cell seeding.

\section{Surface characterization}

A field emission scanning electron microscope (FE-SEM, JSM-6700F) was used to observe the topography of the prepared specimens. The crystallinity of the samples was evaluated by X-ray diffraction (XRD) using a Siemens D-500 diffractometer. Contact angle measurements were carried out by the sessile-drop method with a contact angle measuring system (EasyDrop Standard, KRUSS, Germany) at room temperature. Two different liquids were used to carry out the contact angle measurements: distilled water and diiodomethane. The contact angles $\theta$ were measured by analyzing drop shape using the DSA1 software (KRUSS). From the contact angle results obtained with the two liquids, the surface free energy (SFE) of each surface was calculated. ${ }^{19}$

\section{Cell culture}

Primary rat calvarial osteoblasts were obtained by digestion of one day old Sprague-Dawley rats' calvarial bone. ${ }^{19}$ Ethical approval was granted by the University Research Ethics Committee of the Stomatological Hospital, FMMU (Xi'an, PR China). The cells were cultured in Dulbecco's minimum essential medium (DMEM, Gibco) supplemented with 10\% bovine calf serum (BCS, Gibco) and 1\% penicillin/streptomycin and incubated in a humidified atmosphere of $5 \% \mathrm{CO}_{2}$ at $37^{\circ} \mathrm{C}$. Passages $2-5$ were used in the experiments.

\section{Cell adhesion}

Osteoblasts were seeded on the substrates placed in 24-well culture plate at a density of 17,000 cells $/ \mathrm{cm}^{2}$ and were allowed to attach for 30,60 , and $120 \mathrm{~min}$. At the prescribed time points, the nonadherent cells were removed by rinsing with a phosphate buffered saline (PBS) solution. The cells were then fixed and stained with 4',6'-diamidino-2-phenylindole (DAPI) and the cell numbers were counted in five random fields on each sample under a fluorescence microscope.

\section{Cell viability}

One milliliter of the cell suspension was seeded onto each specimen with a density of $2 \times 10^{4}$ cells $/ \mathrm{ml}$ and were cultured in DMEM with 10\% BCS. After 1, 4, and 7 days postseeding, cell proliferation was assessed using the MTT assay. In brief, at the prescribed time points, specimens were rinsed gently three times with PBS and transferred to a new 24-well culture plate. The MTT solution was added and the specimens were incubated at $37^{\circ} \mathrm{C}$ to allow the formation of formazen. Afterwards, the formazen was dissolved using dimethyl sulfoxide (DMSO) and the optical density (OD) was measured at $490 \mathrm{~nm}$ using a spectrophotometer (Bio-tek, Germany). 
TABLE I. Primers Used for Real-Time PCR

\begin{tabular}{lll}
\hline Gene & Forward Primer Sequence $\left(5^{\prime}-3^{\prime}\right)$ & Reverse Primer Sequence $\left(5^{\prime}-3^{\prime}\right)$ \\
\hline ITG & TGCGATAGGTCCAACGGCTTA \\
RUNX2 & CCATAACGGTCTTCACAAATCCT & CATTTGTCGCTACGCATGGAAC \\
ALP & AACGTGGCCAAGAACATCATCA & TCTGTCTGTGCCTTCTTGGTTC \\
BMP & TAACACCGTGCTCAGCTTCC & TGTCATCTCCAGCCGTGTC \\
OPN & TTCCCACTCATTCTGAAAGTTCC \\
OCN & TCCTGCGGCAAGCATTCTC & CTGCCAAACTCAGCCACTTTCA \\
GAPDH & GGTGCAGACCTAGCAGACACCA & AGGTAGCGCCGGAGTCTATTCA \\
\hline
\end{tabular}

\section{Cell morphology}

The cell morphology was observed using fluorescent staining and SEM. After $3 \mathrm{~h}$ of culture, osteoblasts were fixed in $2 \%$ paraformaldehyde, and stained using Hochest (nuclei blue color, sigma) and rhodamine phalloidin (actin filament green color, sigma) before fluorescence microscopy. After 4 days of culture, the samples with attached cells were washed with PBS and distilled water, fixed, and sputter coated with carbon. The cell morphology was observed using the FE-SEM.

\section{Alkaline phosphatase activity}

The cells were seeded on the substrates in 24-well culture plates at a density of $2 \times 10^{4}$ cells/well. After 7 and 14 days of culture, the cells were washed three times with PBS and lysed in $0.1 \%$ Triton X-100 using four standard freezethaw cycles. The alkaline phosphatase activity (ALP) activities of the samples were determined by a colorimetric assay using an ALP reagent containing p-nitrophenyl phosphate (p-NPP) as the substrate. The absorbance of p-nitrophenol formed was measured at $405 \mathrm{~nm}$. The intracellular total protein content was determined using a commercial BCA protein assay kit and the ALP activity was normalized to the total protein content.

\section{Osteogenesis-related gene expressions}

To investigate the influence of the nanoscale titania surfaces on the gene expressions of seeded osteoblasts, the osteogenisis-related genes were analyzed using the real-time polymerase chain reaction (Real-time PCR). The cells were seeded at $2 \times 10^{4}$ cells/well and cultured for 3 and 10 days. The total RNA was isolated using the TRIzol reagent (Gibco). One microgram of RNA from each sample was reverse transcribed into complementary DNA (cDNA) using the PrimeScript ${ }^{\mathrm{TM}}$ RT reagent kit (TaKaRa). The forward and reverse primers for the selected genes are listed in Table I. Expression of osteogenesis-related genes including integrin- $\beta 1$ (ITG), runt-related transcription factor 2 (RUNX2), ALP, bone morphogenetic protein-2 (BMP), osteopontin (OPN), and osteocalcin (OCN) were quantified using the Real-time PCR (Bio-Rad $\mathrm{iQ}^{\mathrm{TM}} 5$ Multicolor Real-Time PCR Detection System) with SYBR ${ }^{\circledR}$ Premix Ex ${ }^{\mathrm{TM}}$ Taq II (TaKaRa). Data analysis was carried out using the $\mathrm{iQ}^{\mathrm{TM}_{5}}$ Optical System Software Version 2.0 (Bio-Rad). The relative expression levels for each gene of interest were normalized to that of the housekeeping gene GAPDH.

\section{Statistical analysis}

The data were analyzed using SPSS 14.0 software (SPSS, USA). A one-way ANOVA followed by a Student-NewmanKeuls post hoc test was used to determine the level of significance. $p<0.05$ was considered to be significant and $p<$ 0.01 was considered to be highly significant.

\section{RESULTS}

\section{Titania nanotube surface characterization}

In the SEM micrographs depicted in Figure 1, the nanostructured surface is evident on the anodized titanium. A nanonet-like texture is formed at $5 \mathrm{~V}$ and the average diameter of the holes is about $25 \mathrm{~nm}$. At $20 \mathrm{~V}$, a nanotubular texture is formed and the typical dimension of the hollow titania nanotubes is about $80 \mathrm{~nm}$ in inner diameter. The XRD patterns show that the titania nanotube surface after sterilization have a amorphous nature (data not shown). The contact angles and values of surface free energy are listed in Table II. The anodization treatment dramatically decreases the water contact angle on titania and enhances the surface free energy, especially the polar component. Anodization at $20 \mathrm{~V}$ results in a little smaller water contact angle and higher surface energy than at $5 \mathrm{~V}$.

\section{Cell adhesion and cell viability}

No significant difference among the adherent cell numbers on the 5 and $20 \mathrm{~V}$ anodized surfaces and polished surface is observed after 30,60, and $120 \mathrm{~min}$ of culture (Fig. 2). At days 1,4 , and 7 , there is still no large difference for the cell viability on the 5 and $20 \mathrm{~V}$ anodized surfaces and the polished surface, except that the cell proliferation on the $5 \mathrm{~V}$

TABLE II. Contact Angles $\left({ }^{\circ}\right)$ and Values of Surface Free Energy $\left(\mathrm{mJ} / \mathrm{m}^{2}\right)$ of Specimens

\begin{tabular}{|c|c|c|c|c|c|}
\hline \multirow[b]{2}{*}{ Samples } & \multicolumn{2}{|c|}{ Contact Angle $\left({ }^{\circ}\right)$} & \multicolumn{3}{|c|}{ Surface Free Energy $\left(\mathrm{mJ} / \mathrm{m}^{2}\right)$} \\
\hline & Distilled Water & Diiodomethane & Total & Polar & Dispersive \\
\hline $5 \mathrm{~V}$ & $31.18 \pm 3.92$ & $19.70 \pm 3.26$ & 72.22 & 24.35 & 47.87 \\
\hline $20 \mathrm{~V}$ & $25.39 \pm 4.31$ & $14.57 \pm 2.84$ & 75.36 & 26.18 & 49.18 \\
\hline$P$ & $61.54 \pm 4.46$ & $33.44 \pm 1.36$ & 53.31 & 10.58 & 42.74 \\
\hline
\end{tabular}



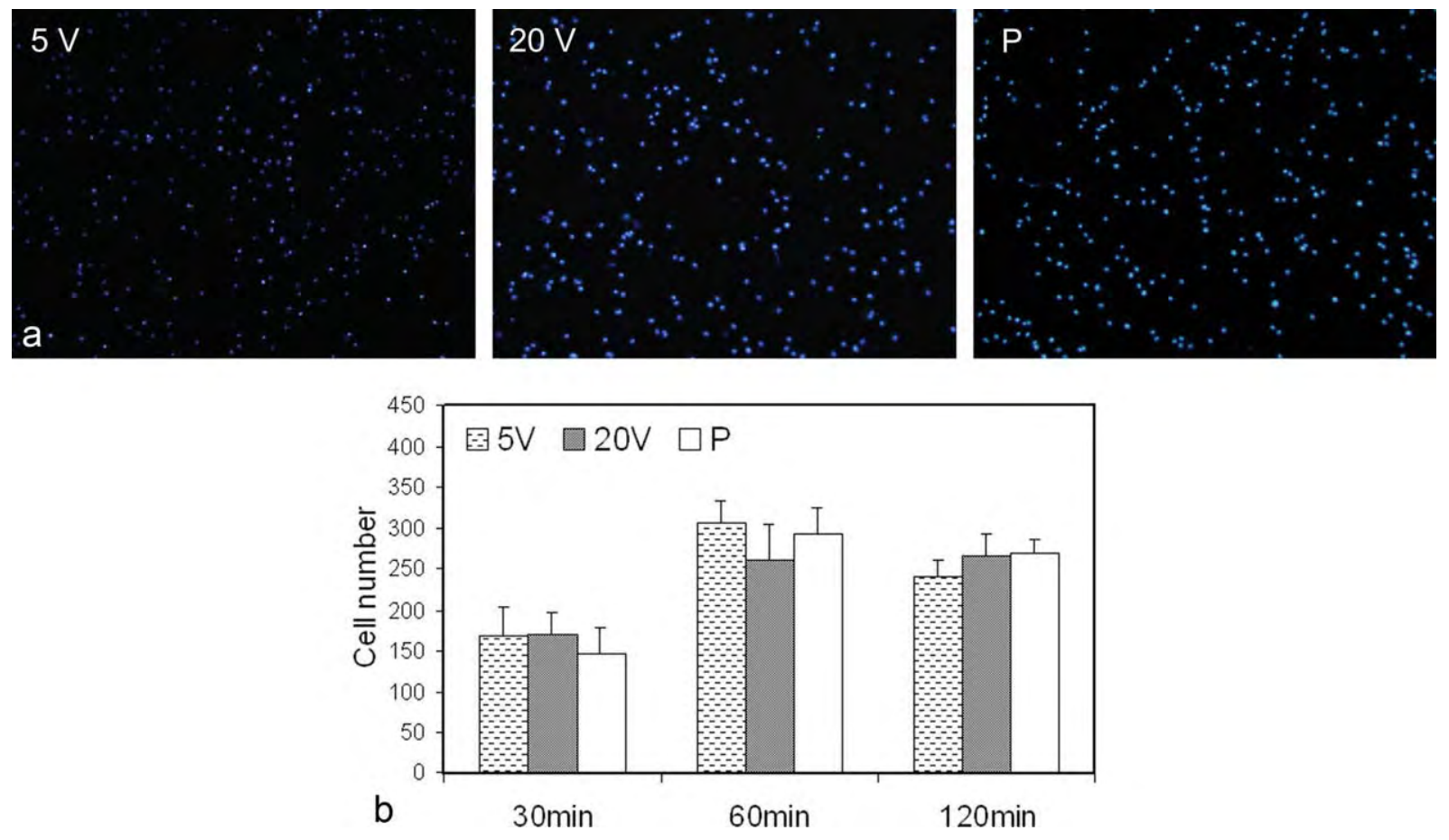

FIGURE 2. (a) Fluorescence micrographs of osteoblasts (nuclei in blue) incubated on samples for 60 min of culture and (b) Osteoblasts adhesion measured by cell counting at 30,60 , and $120 \mathrm{~min}$. [Color figure can be viewed in the online issue, which is available at wileyonlinelibrary.com.]

anodized surface is a little lower than that on the other two titania surfaces at days 1 and 4 (Fig. 3).

\section{Cell morphology}

Osteoblasts grow and spread well on all three surfaces after $3 \mathrm{~h}$ or 4 days of culture (Figs. 4 and 5). The SEM pictures show that the cells spread well and attach closely onto all three substrates (Fig. 4). In particular, on the $20 \mathrm{~V}$ anodized surface, the cells seem to show a larger amount of thin fillopodia [Figs. 4(b,e)]. This phenomenon is already obvious after $3 \mathrm{~h}$ of culture as shown by the fluorescence pictures. On the $20 \mathrm{~V}$ surface, many more cells show a large amount of microfilament compared with other two surfaces (Fig. 5). The higher magnification SEM pictures disclose that the nanoscale extracellular matrix (ECM) deposited on the ano-

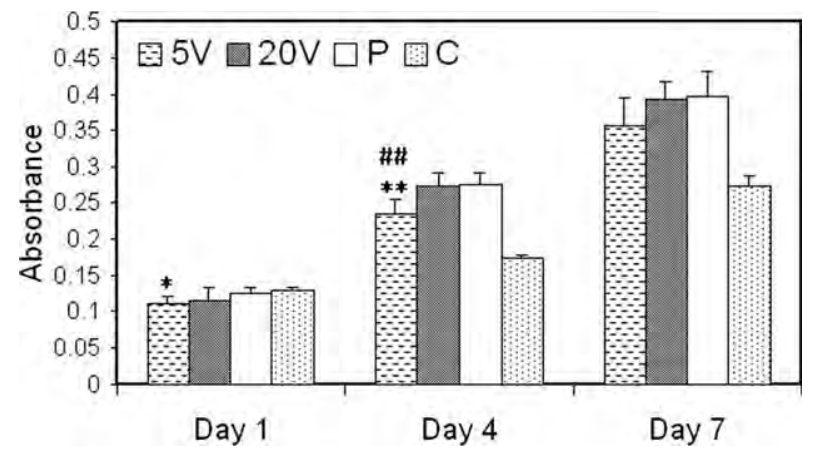

FIGURE 3. Cell viability/proliferation on samples after 1, 4, and 7 days of incubation using colorimetric MTT assay. ${ }^{*} p<0.05$ and ${ }^{*} p<0.01$ compared with the polished surface and ${ }^{\# \#} p<0.01$ compared with the $20 \mathrm{~V}$ surface. dized surfaces follows the surface texture [Figs. 4(g,h)], showing forms of nanopartilces on the $5 \mathrm{~V}$ surface with a diameter of about $30 \mathrm{~nm}$ and nanobelts on the top edge of nanotube with a width of about $20 \mathrm{~nm}$. On the polished surface, less ECM is deposited [Fig. 4(i)].

\section{Intracellular alkaline phosphatase activity}

Even without the osteogenic medium, the primary osteoblasts on the samples still differentiate and synthesize ALP (Fig. 6). At day 7, the ALP activity of the osteoblasts on the $20 \mathrm{~V}$ surface is higher than that on the polished surface, while that on the $5 \mathrm{~V}$ surface is lower than that on the control. After 14 days of culture, the osteoblasts on the substrates synthesize more ALP. The ALP activity of the osteoblasts on the $5 \mathrm{~V}$ and $20 \mathrm{~V}$ anodized surfaces is higher than that on the polished surface but with no statistical significance.

\section{Osteogenesis-related gene expression}

The gene expression on different substrates is quantified using Real-time PCR and the results are compared in Figure 7. In general, the osteoblasts cultured on the anodized surfaces exhibit down-regulated gene expressions. After 3 days of culture, both anodized nanotextured surfaces induce downregulation expressions of nearly all the genes concerned in this study, although statistical differences are found only in the expressions of RUNX2 and OPN. On the three surfaces, the expressions of all the genes show a time-dependent increase. After incubation for 10 days, there are no obvious differences between the osteoblast gene expressions on the $5 \mathrm{~V}$ anodized surface and the polished control surface. However, the expressions of ITG, RUNX2, BMP, and OPN on the 

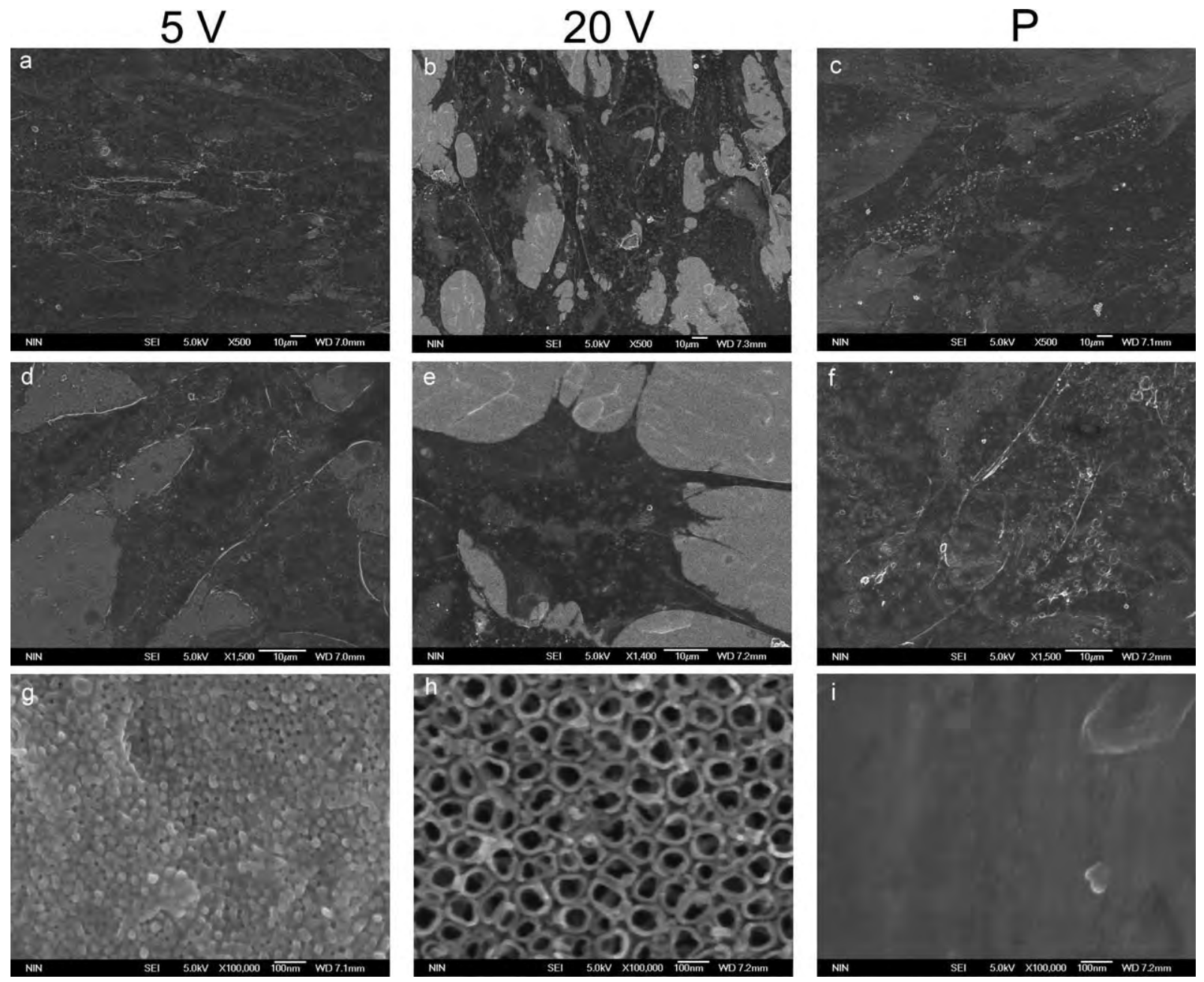

FIGURE 4. Osteoblast cell morphology and ECM deposition on the $5 \mathrm{~V}$ anodized surface (a, $\mathrm{d}$, and $\mathrm{g}$ ), the $20 \mathrm{~V}$ anodized surface (b, e, and h) and the polished surface (c, $f$, and i) after 4 days of culture.

$20 \mathrm{~V}$ anodized surface are statistically lower than those on the other two surfaces.

\section{DISCUSSION}

Titania nanotubes have attracted much attention recently for their potential in implant application. However, the biological behavior of titania nanotubes is still controversial. $^{5,6,8,9}$ Considering that the biological behavior of primary osteoblasts is closer to the situation in vivo and that different phenotypes of osteoblasts used in previous studies may account for the controversial results, we monitor the behavior of primary osteoblasts on the nanoscale titania surfaces fabricated by anodization. It should be noted that these samples are not annealed but they are sterilized by ultraviolet irradiation. These factors may affect the experimental results.

Two distinct surfaces, the $5 \mathrm{~V}$ anodized surface with a pore diameter of $25 \mathrm{~nm}$ and the $20 \mathrm{~V}$ anodized surface with an inner tubular diameter of $80 \mathrm{~nm}$ are formed by anodization. These two surfaces are chosen to observe the pore size effect on primary osteoblasts. The XRD results show that the surfaces have an amorphous nature. The different phases of titania, amorphous, anatase, and rutile, influence the cell functions on them. ${ }^{20}$ The surface free energy is considered to play an important role in the cell/implant interaction $^{21}$ and so it is monitored in this study. The anodization treatment enhances the surface free energy of titanium and the surface energy of the $20 \mathrm{~V}$ surface is a little higher than that of the $5 \mathrm{~V}$ surface. However, in our study, the cell behavior cannot be explained well by the surface free energy difference. Higher surface free energy is believed to benefit cell function, but in this study the anodized surfaces with higher surface free energy induce down-regulated gene expressions. The surface nanotopography may thus play a more important role than the surface free energy, which has also been indicated by other study. ${ }^{22}$ It is worth mentioning that there is residual fluoride in the nanotubes resulting from the preparation procedure. ${ }^{20}$ Proper content of fluoride is widely reported to encourage osteoblast function, ${ }^{20}$ so the down-regulation of gene expressions cannot be attributed to the fluoride residual.

Initial adhesion of anchorage dependent cells is critical for their subsequent functions. ${ }^{23}$ We observe no obvious difference in the initial cell adhesion on the different 

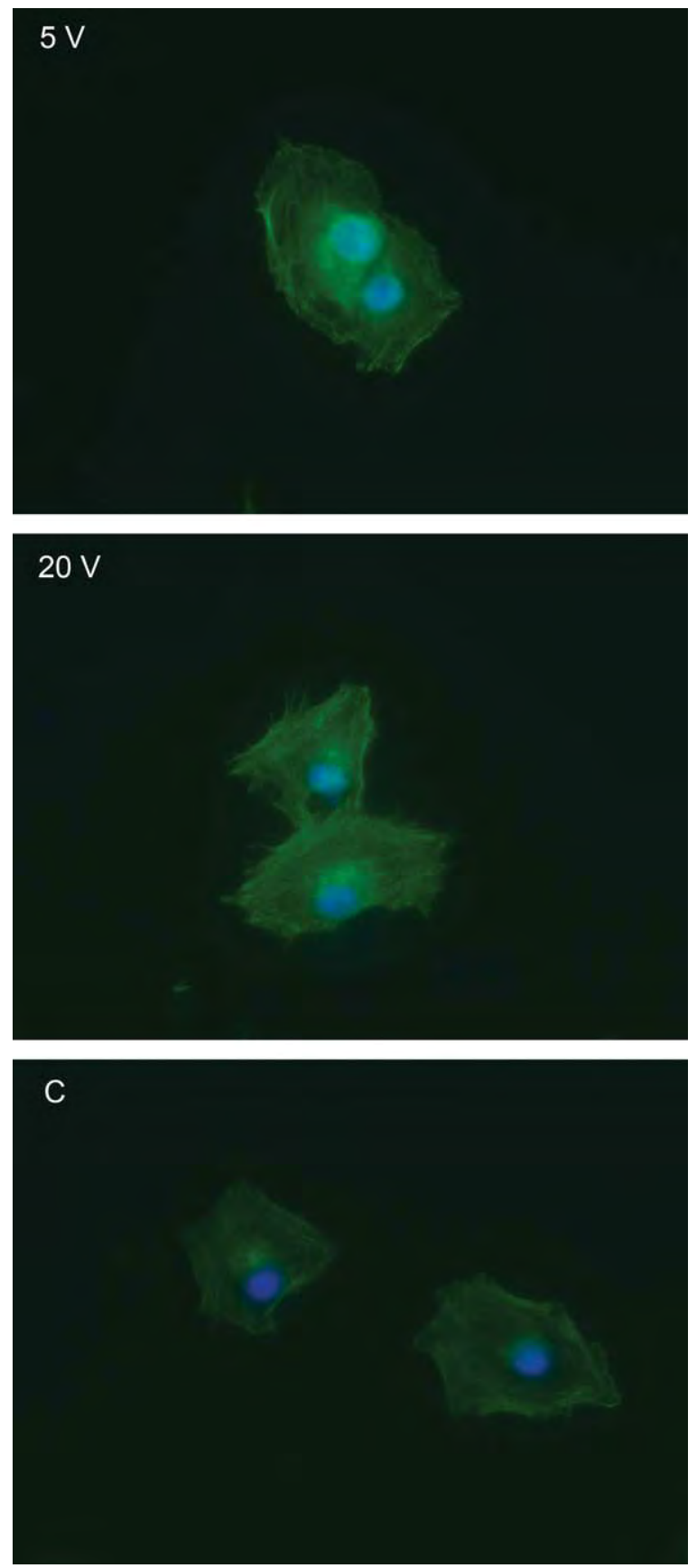

FIGURE 5. Fluorescence microscopy images of the cells with dual staining with phalloidin for actin filaments (green) and Hochest for nuclei (blue). [Color figure can be viewed in the online issue, which is available at wileyonlinelibrary.com.]

substrates. Our observation is somewhat similar to those reported previously that no difference of initial cell attachment can be found in the initial 2 h., ${ }^{6,8,18}$ However, after one week, controversial results have been reported. Some have observed enhanced growth of MC3T3-E1 on titania nanotubes, ${ }^{6,8}$ while others have reported inhibited growth of primary human osteoblast-like cells on titania nanotubes except the $15 \mathrm{~nm}$ ones. ${ }^{5}$ Besides the substantially different nature of the titania nanotubes in these studies, we think that the different phenotypes of the osteoblasts used in their studies may also be responsible for the different results. Our studies are partially consistent with Park et al.'s results showing that growth of primary osteoblasts on the anodized nanotextured surfaces with tube diameters larger than $15 \mathrm{~nm}$ is a little slower than that on the control. ${ }^{5}$ The differential growth behavior of the primary osteoblasts and osteoblastic cell lines may be explained by the differences in their biological behavior. The osteoblastic cell lines show faster proliferation speed and abnormal differentiation phenotype in contrast with the primary cells during culture. ${ }^{24}$ It has been reported that the groove texture MC3T3-E1 cells are only slightly affected by the groove texture, whereas primary rat bone marrow cell are affected more significantly. ${ }^{15}$ Hence, it is reasonable that the osteoblastic cell line and the primary osteoblast may react differently to the nanoscale textures.

SEM reveals that the cells attach closely to the polished surface and are connected with each other nearly fully covering the entire substrate. The cells spread out with robust lamellopodia on the $25 \mathrm{~nm}$ nanonet-like surface whereas the cells on the $80 \mathrm{~nm}$ nanotubes show a large amount of thin fillopodia. This phenomenon has been observed in previous study for osteoblasts ${ }^{5,6,8,18}$ and other cells such as mesenchymal stem cells ${ }^{4,25}$ and endothelial cells. ${ }^{26}$ This long fillopodia may be ascribed to the poor focal contact formation on the nanotubular surfaces. ${ }^{4,5,25}$ On the titania nanotubes with a larger dimension, the ECM is mainly deposited on the edge of the nanotubes [Fig. 4(h)], thus providing insufficient ECM attachment sites for the cells. This have been partly verified by Lee's study, in which cells form long fillopodia when they are on a substrate not suitable for focal contact formation (silicon dioxide nanorods in that study) and trying to find a more suitable area to attach and proliferate. $^{22}$

A higher ALP activity is observed on the anodized surfaces even though a lower activity is seen on the $5 \mathrm{~V}$ surface during the first 1 week of culture. This is more or less in accordance with ALP gene expression pattern obtained by real-time PCR. Others have reported an elevated ALP activity of MC3T3-E1 on the nanotubular surfaces compared

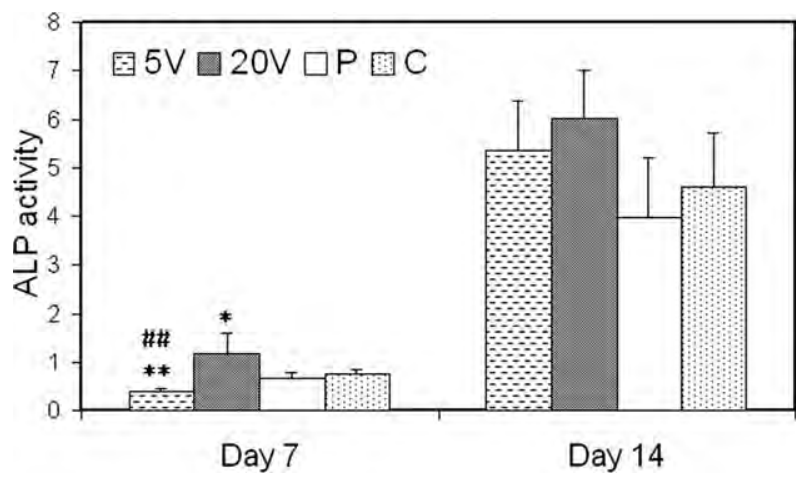

FIGURE 6. ALP activity of osteoblasts on samples after 7 and 14 days of culture. ${ }^{*} p<0.05$ and ${ }^{* *} p<0.01$ compared with the polished surface and ${ }^{\# \#} p<0.01$ compared with the $20 \mathrm{~V}$ surface. 

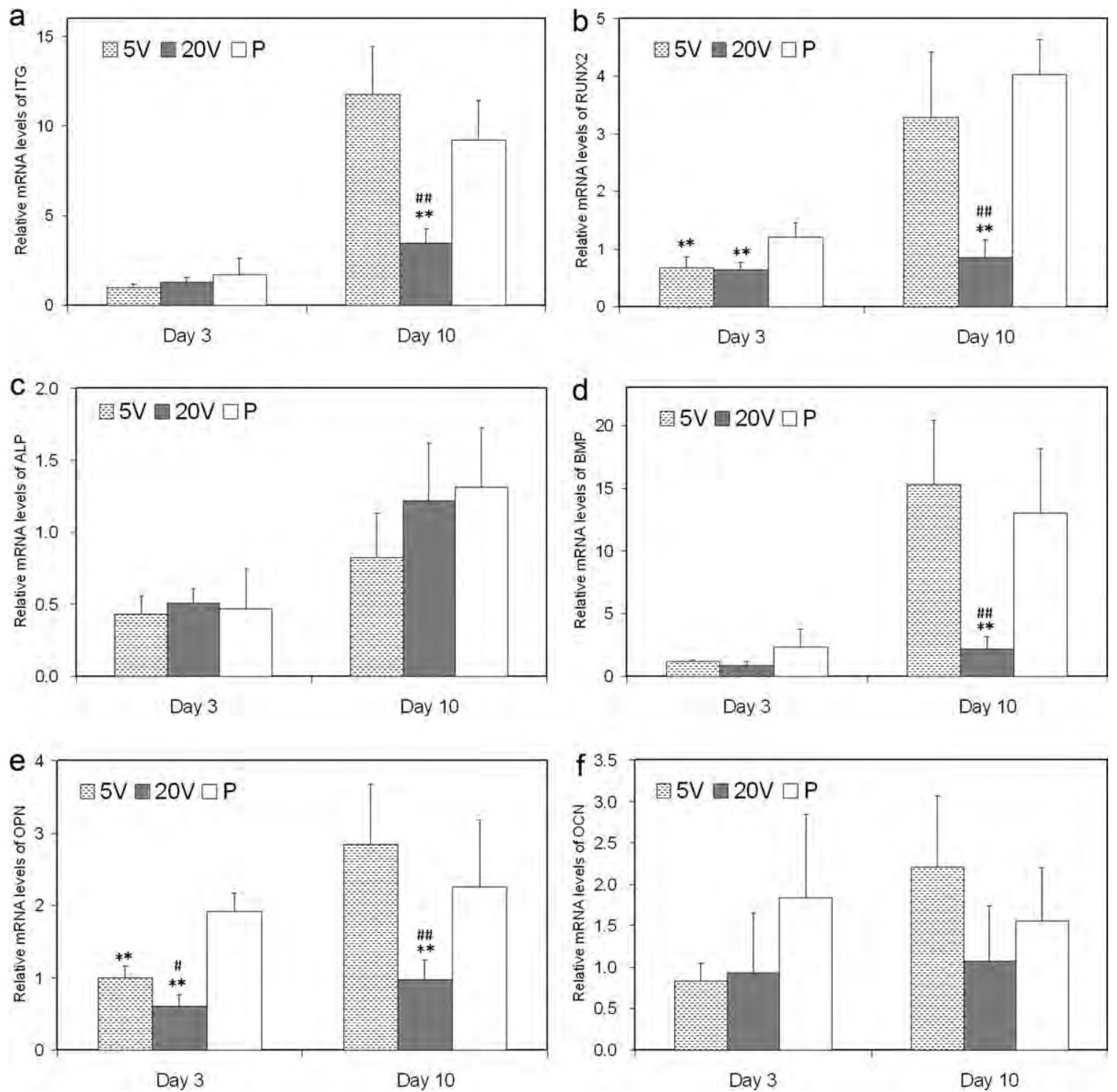

FIGURE 7. Expression of osteogenesis-related genes by primary osteoblasts cultured on titanium surfaces after incubation for 3 and 10 days: (a) Integrin- $\beta 1$, (b) RUNX2, (c) ALP, (d) BMP, (e) osteopontin, and (f) osteocalcin. The data were generated by Real-time RT-PCR and were shown as mean expression relative to the housekeeping gene GAPDH $\pm \mathrm{SD}$. ${ }^{*} p<0.05$ and ${ }^{* *} p<0.01$ compared with the polished surface, ${ }^{*} p<0.05$ and $\#$ \# $p$ 0.01 compared with the $5 \mathrm{~V}$ anodized surface.

with titania surfaces with the complete media. ${ }^{9}$ The exact reason for the elevated ALP activity on the nanotubular surface as well as the tendency with MC3T3-E1 is still unclear but it is believed that the higher cell mobility on the titania nanotube may play a part.

To the best of our knowledge, it is the first time that down-regulated expression of osteogenesis-related genes of primary osteoblasts incubated on anodized nanotextures is reported. Gene expressions are severely impaired on the $20 \mathrm{~V}$ anodized surface compared with the $5 \mathrm{~V}$ one. Cell adhesion on biomaterials and associated signal transduction are mediated by focal adhesion involving integrin clustering. More and more evidences indicate that clustering of integrins requires that the spacing between ligated integrins be less than $50-70 \mathrm{~nm} .{ }^{27}$ Park et al. suggest that a spacing of
$15 \mathrm{~nm}$ may provide the optimum length scale for integrin clustering and focal contact formation and spacings larger than $50 \mathrm{~nm}$ severely hamper them. ${ }^{4}$ The down-regulated gene expressions on the anodized surfaces may be related to hampered focal adhesion formation and it is more severely impaired on the $20 \mathrm{~V}$ anodized surface for the larger tube size of $80 \mathrm{~nm}$, leading to much lower gene expressions. The down-regulated expression of Itegrin- $\beta 1$ may confirm this hypothesis. Itegrin- $\beta 1$ is associated with the integrins expressed by osteoblasts representing the dominant adhesion mechanism to $\mathrm{ECM}^{28}$ The signals from the ECM transduced through the focal contacts into the cells are very important for cell proliferation, differentiation, or apoptosis. $^{29}$ The shortage of signals from ECM for inadequate focal contact formation on the anodized surfaces, especially the 
$20 \mathrm{~V}$ one, results in checked cell differentiation and associated gene expressions. Thereafter, it is reasonable that the expression of RUNX2 which is an important transcription factor that contributes to the regulation of the expression of many osteoblast genes ${ }^{30}$ and the downstream genes such as BMP, OPN, and OCN are down-regulated. Recently Biggs et al. have shown that the nanopit arrays (100 $\mathrm{nm}$ deep and $120 \mathrm{~nm}$ in diameter possessing a centre-centre spacing of $300 \mathrm{~nm}$ ) disrupt adhesion formation and cellular spreading in primary human osteoblasts preventing osteospecific differentiation in human osteoprogenitors. ${ }^{31}$ It supports our results to a certain degree.

\section{CONCLUSION}

We observe depressed functions of primary osteoblasts on the anodized nanotextured surfaces. Initial cell adhesion is not obviously affected by the anodized surfaces. A little higher intracellular ALP activity and more ECM deposition are found on the anodized surfaces, while cell growth and osteogenesis-related gene expression are both impaired on them, especially the $20 \mathrm{~V}$ anodized surface. This may be attributed to the compromised focal contact formation on the anodized surfaces. The difference in the phenotypes of the primary osteoblasts and osteoblastic cell lines may account for previous conflicting reports in osteoblast cytocompatibility of titania nanotubes. It should be considered when evaluating the biocompatibility of titania nanotubes as well as comparing results from different sources.

\section{REFERENCES}

1. Webster TJ, Ejiofor JU. Increased osteoblast adhesion on nanophase metals: Ti, Ti6Al4V, and CoCrMo. Biomaterials 2004;25: 4731-4739.

2. Hajicharalambous CS, Lichter J, Hix WT, Swierczewska M, Rubner MF, Rajagopalan P. Nano- and sub-micron porous polyelectrolyte multilayer assemblies: Biomimetic surfaces for human corneal epithelial cells. Biomaterials 2009;30:4029-4036.

3. Mendonca G, Mendonca DB, Aragao FJ, Cooper LF. Advancing dental implant surface technology-from micron- to nanotopography. Biomaterials 2008;29:3822-3835.

4. Park J, Bauer S, von der Mark K, Schmuki P. Nanosize and vitality: $\mathrm{TiO}_{2}$ nanotube diameter directs cell fate. Nano Lett 2007;7:1686-1691.

5. Park J, Bauer S, Schlegel KA, Neukam FW, von der Mark K, Schmuki P. $\mathrm{TiO}_{2}$ Nanotube Surfaces: $15 \mathrm{~nm}$-An optimal length scale of surface topography for cell adhesion and differentiation. Small 2009;5:666-671.

6. Brammer KS, Oh S, Cobb CJ, Bjursten LM, Heyde HV, Jin S. Improved bone-forming functionality on diameter-controlled $\mathrm{TiO}_{2}$ nanotube surface. Acta Biomater 2009;5:3215-3223.

7. Oh SH, Finones RR, Daraio C, Chen LH, Jin SH. Growth of nanoscale hydroxyapatite using chemically treated titanium oxide nanotubes. Biomaterials 2005;26:4938-4943.

8. Oh S, Daraio C, Chen LH, Pisanic TR, Finones RR, Jin S. Significantly accelerated osteoblast cell growth on aligned $\mathrm{TiO}_{2}$ nanotubes. J Biomed Mater Res A 2006;78:97-103.

9. Popat KC, Eltgroth M, Latempa TJ, Grimes CA, Desai TA. Decreased Staphylococcus epidermis adhesion and increased osteoblast functionality on antibiotic-loaded titania nanotubes. Biomaterials 2007;28:4880-4888.
10. Das $\mathrm{K}$, Bose $\mathrm{S}$, Bandyopadhyay $\mathrm{A} \cdot \mathrm{TiO}_{2}$ nanotubes on Ti: Influence of nanoscale morphology on bone cell-materials interaction. J Biomed Mater Res A 2009;90:225-237.

11. Balasundaram G, Yao $\mathrm{C}$, Webster $\mathrm{TJ}$. $\mathrm{TiO}_{2}$ nanotubes functionalized with regions of bone morphogenetic protein-2 increases osteoblast adhesion. J Biomed Mater Res A 2008;84:447-453.

12. Eaninwene G II, Yao C, Webster TJ. Enhanced osteoblast adhesion to drug-coated anodized nanotubular titanium surfaces. Int $\mathrm{J}$ Nanomed 2008;3:257-264.

13. Crawford GA, Chawla N, Das K, Bose S, Bandyopadhyay A. Microstructure and deformation behavior of biocompatible $\mathrm{TiO}_{2}$ nanotubes on titanium substrate. Acta Biomater 2007;3:359-367.

14. Bjursten LM, Rasmusson L, Oh S, Smith GC, Brammer KS, Jin S. Titanium dioxide nanotubes enhance bone bonding in vivo. J Biomed Mater Res A 2010;92:1218-1224.

15. Bruinink A, Wintermantel E. Grooves affect primary bone marrow but not osteoblastic MC3T3-E1 cell cultures. Biomaterials 2001;22: 2465-2473.

16. Rausch-fan X, Qu Z, Wieland M, Matejka M, Schedle A. Differentiation and cytokine synthesis of human alveolar osteoblasts compared to osteoblast-like cells (MG63) in response to titanium surfaces. Dent Mater 2008;24:102-110.

17. Peng L, Eltgroth ML, LaTempa TJ, Grimes CA, Desai TA. The effect of $\mathrm{TiO}_{2}$ nanotubes on endothelial function and smooth muscle proliferation. Biomaterials 2009;30:1268-1272.

18. Oh S, Jin S. Titanium oxide nanotubes with controlled morphology for enhanced bone growth. Mater Sci Eng C 2006;26: 1301-1306.

19. Zhao L, Wei Y, Li J, Han $Y$, Ye R, Zhang Y. Initial osteoblast functions on Ti-5Zr-3Sn-5Mo-15Nb titanium alloy surfaces modified by microarc oxidation. J Biomed Mater Res A 2010;92:432-440.

20. Park J, Bauer S, Schmuki P, von der Mark K. Narrow window in nanoscale dependent activation of endothelial cell growth and differentiation on $\mathrm{TiO}_{2}$ nanotube surfaces. Nano Lett 2009;9: 3257-3164.

21. Kennedy SB, Washburn NR, Simon CG Jr, Amis EJ. Combinatorial screen of the effect of surface energy on fibronectin-mediated osteoblast adhesion, spreading and proliferation. Biomaterials 2006:27:3817-3824.

22. Lee J, Chu BH, Chen $\mathrm{KH}$, Ren F, Lele TP. Randomly oriented, upright $\mathrm{SiO}_{2}$ coated nanorods for reduced adhesion of mammalian cells. Biomaterials 2009;30:4488-4493.

23. Anselme K. Osteoblast adhesion on biomaterials. Biomaterials 2000;21:667-681.

24. Clover J, Gowen M. Are MG-63 and HOS TE85 human osteosarcoma cell lines representative models of the osteoblastic phenotype? Bone 1994;15:585-591.

25. Oh S, Brammer KS, Li YSJ, Teng D, Engler AJ, Chien S, Jin S. Stem cell fate dictated solely by altered nanotube dimension. Proc Natl Acad Sci USA 2009;106:2130-2135.

26. Brammer KS, Oh SH, Gallagher JO, Jin SH. Enhanced cellular mobility guided by $\mathrm{TiO}_{2}$ nanotube surfaces. Nano Lett 2008;8: 786-793.

27. Cavalcanti-Adam EA, Volberg T, Micoulet A, Kessler H, Geiger B, Spatz JP. Cell spreading and focal adhesion dynamics are regulated by spacing of integrin ligands. Biophys J 2007;92:2964-2974.

28. Garcia AJ. Get a grip: Integrins in cell-biomaterial interactions. Biomaterials 2005;26:7525-7529.

29. Hynes RO. Integrins: Bidirectional, allosteric signaling machines. Cell 2002;110:673-687.

30. Ducy P. Cbfa1: A molecular switch in osteoblast biology. Dev Dyn 2000;219:461-471.

31. Biggs MJP, Richards RG, Gadegaard N, Wilkinson CDW, Oreffo ROC, Dalby MJ. The use of nanoscale topography to modulate the dynamics of adhesion formation in primary osteoblasts and ERK/MAPK signalling in STRO $-1^{+}$enriched skeletal stem cells. Biomaterials 2009:30:5094-5103. 